\title{
Varicella vaccination during early pregnancy: a cause of in utero miliary fetal tissue calcifications and hydrops?
}

\author{
Joseph Apuzzio, Vijaya Ganesh, Leslie Iffy and Anthony Al-Khan \\ Department of Obstetrics, Gynecology and Women's Health, New Jersey Medical School, Newark, NJ
}

\begin{abstract}
Background: It is the purpose of this article to describe a suspected association of inadvertent vaccination with varicella vaccine during early pregnancy with the subsequent development of in utero miliary fetal tissue calcifications and fetal hydrops detected by sonogram at 15 weeks of gestation.

Case: This is a case presentation of a pregnant patient who received varicella vaccination during the same menstrual cycle that she became pregnant, and is supplemented by a literary review. The fetus developed miliary fetal tissue calcifications and fetal hydrops detected by a targeted sonogram at 15 weeks gestation.

Conclusion: Varicella vaccination during early pregnancy may be a cause of miliary fetal tissue calcifications and fetal hydrops.
\end{abstract}

Key words: IMMUNIZATION; COMPLICATION; VIRAL

Varicella vaccine is a live attenuated viral vaccine that was licensed and became available in May 1995. This article describes a suspected association of varicella vaccination given close to the time of conception with the development of miliary fetal tissue calcification and fetal hydrops.

\section{CASE}

The patient is a 30 -year-old gravida 1 para 0 , nursing student, who, because she was varicella susceptible, and as a requirement for her nursing studies, was administered varicella vaccine about 2 weeks after her last normal menstrual period. The patient missed the next menstrual period and was subsequently determined to be pregnant. The family history of both partners was negative for medical and genetic problems. The patient was sent for genetic counseling and a targeted sonogram at approximately 15 weeks gestation. The fetal biometric measurements were compatible with gestational age. However, there were multiple echodensities in the liver and kidneys of the fetus which had the same echodensity as bone (Figure 1). There was also mild subcutaneous edema of the abdomen seen but no ascites. The patient was offered amniocentesis for polymerase

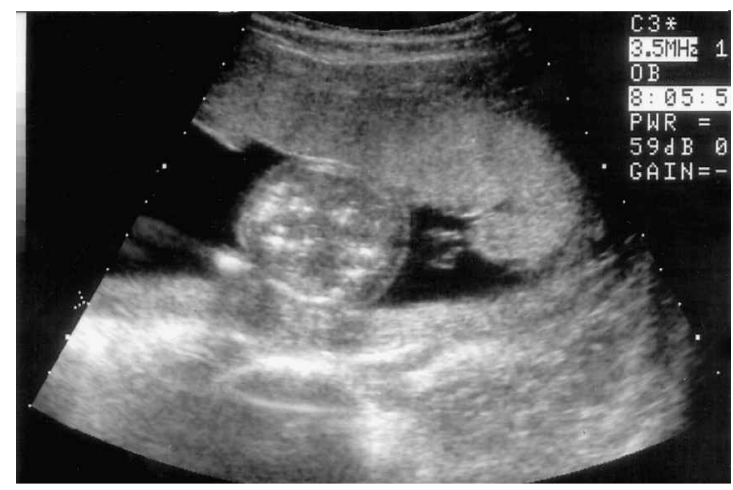

Figure I Sonogram of cross-section of fetal abdomen showing subcutaneous edema and echodensities in fetal liver 
chain reaction (PCR) testing of the fluid for varicella but declined. In view of the fetal tissue calcifications and the fetal hydrops, the patient elected to terminate the pregnancy. At the time of the termination, she declined an autopsy of the fetus and she also declined enrollment in the varicella vaccine registry. Maternal serology for both IgG and IgM for toxoplasmosis, cytomegalovirus, and parvovirus B19 were negative. Herpes simplex virus (HSV) IgM was negative. The venereal disease research laboratory test for syphilis was negative and rubella immune. The blood type was O positive, and the antibody screen was negative.

\section{DISCUSSION}

Although there is no definitive diagnosis in this case because the patient declined amniocentesis for varicella PCR studies and an autopsy of the fetus and analysis of the fetal tissue for the varicella virus, it appears that the miliary fetal tissue calcifications were possibly due to the varicella vaccination administered during the cycle in which the patient became pregnant. Other potential causes for fetal tissue calcifications, including acute infection with a TORCH organism, syphilis, and HSV, as well as the genetic history were negative.

Hartung and colleagues described a fetus with remarkably similar sonographic findings of miliary calcifications due to community acquired varicella virus infection during pregnancy ${ }^{1,2}$. They confirmed fetal infection with varicella zoster virus (VZV) DNA in the fetal blood and in the amniotic fluid tested by PCR. Following abortion fetal infection was confirmed by the detection of VZV in several fetal tissues and the placenta, and also by histopathologic findings presenting as miliary calcified necrosis in the fetal organs.

We attempted to enroll the patient into the pregnancy registry for the varicella vaccine, but since the patient declined to sign consent for enrollment, the case was never enrolled. However, the registry accumulates reported cases and issues an annual report. The latest annual report is a review of information of pregnancies received from May 1995 to December 1998. Of the outcomes of completed pregnancies, there is no report of fetal liver calcifications and hydrops. There were six prospectively reported cases with congenital abnormalities out of 257 reported live births, for a rate of 2.3/100. To date, there has been no reported cases of congenital varicella syndrome post immunization.

We recommend that any patient who has received varicella vaccination shortly before or during pregnancy be counseled about the risks of exposure. Furthermore, the patient should have a targeted sonogram and appropriate follow-up including amniocentesis for confirmative diagnosis if the sonogram is abnormal. In order to obtain a valid database for the analysis of fetal risk, any patient who was vaccinated, either just before or during a pregnancy, should be reported irrespective of a finding of a fetal congenial anomaly or not.

\section{REFERENCES}

1. Hartung J, Enders G, Chaoui R, et al. Prenatal diagnosis of congenital varicella syndrome and detection of VZ virus in the fetus: a case report. Perinatal Diag 1999;19:163-6

ReCEIVED 02/07/02; ACCEPTED 05/29/02
2. Mustonen K, Mustakangas P, Valanne L, et al. Congenital varicella zoster virus infection after maternal subclinical infection: clinical and neuropathological findings. J Perinatol 2001;21:141-6 


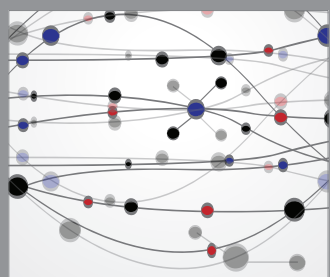

The Scientific World Journal
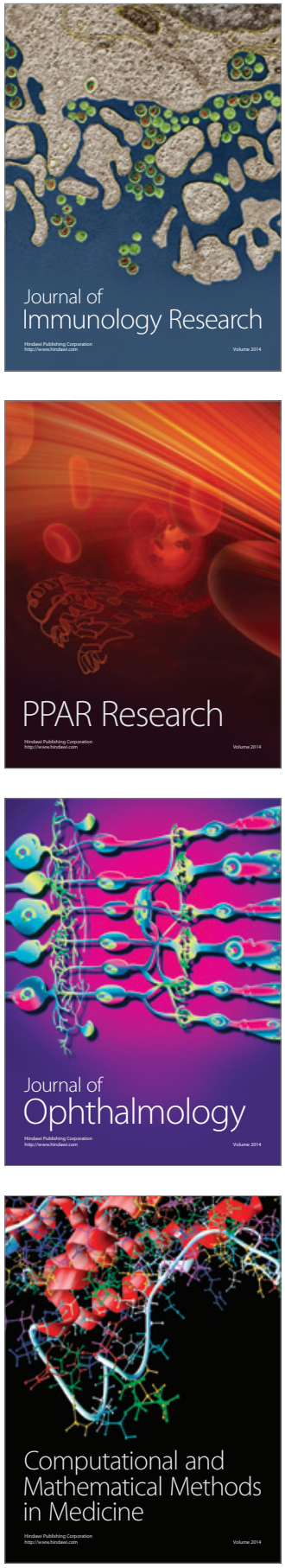

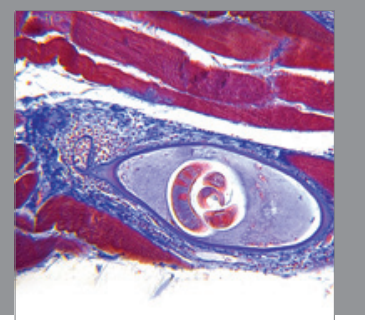

Gastroenterology

Research and Practice
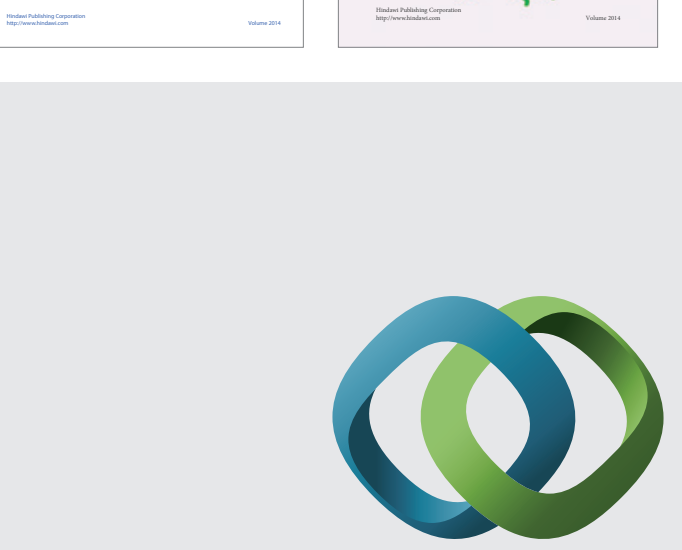

\section{Hindawi}

Submit your manuscripts at

http://www.hindawi.com
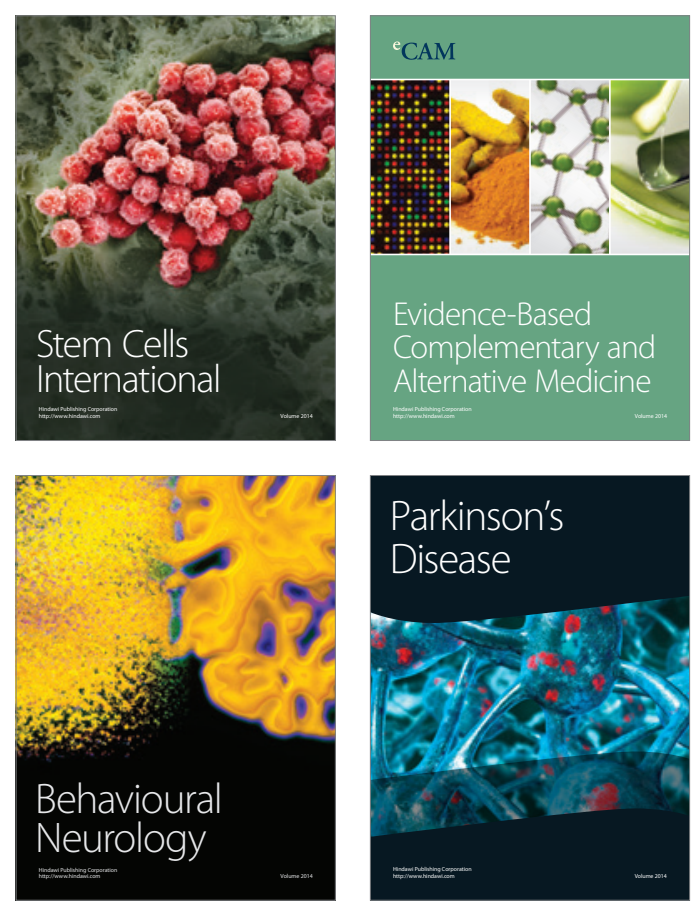

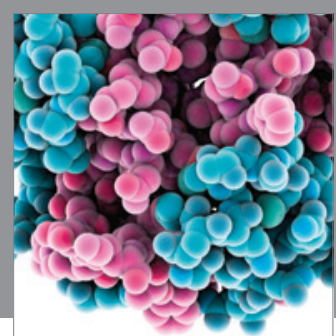

Journal of
Diabetes Research

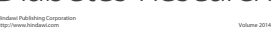

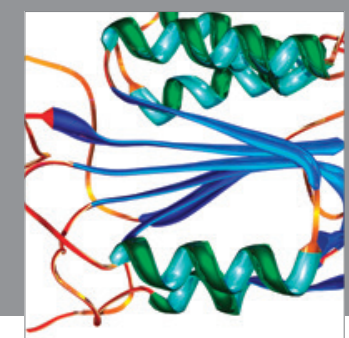

Disease Markers
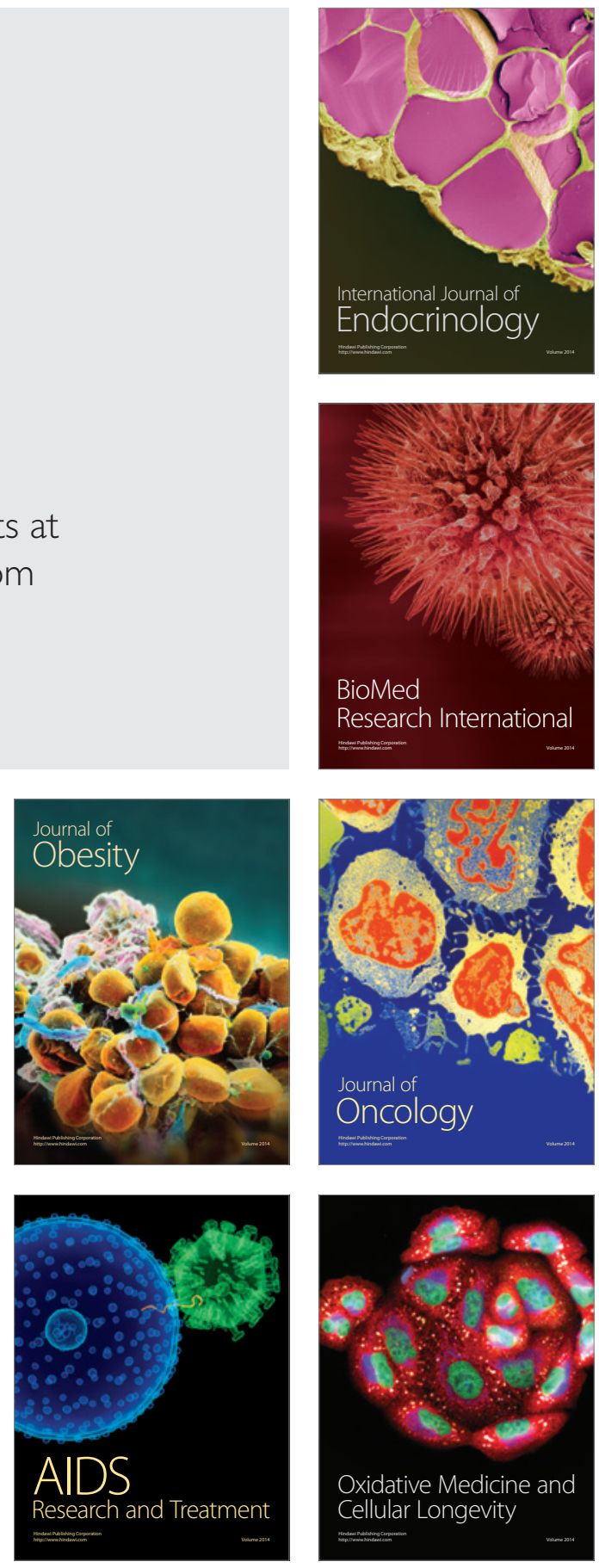